\title{
Dual Ultrastructural Localization of Enkephalin and Tyrosine Hydroxylase Immunoreactivity in the Rat Ventral Tegmental Area: Multiple Substrates for Opiate-Dopamine Interactions
}

\author{
Susan R. Sesack and Virginia M. Pickel \\ Division of Neurobiology, Department of Neurology and Neuroscience, Cornell University Medical College, New York, New \\ York 10021
}

Endogenous opiates modulate activity in the mesocorticolimbic dopaminergic system, and this interaction is thought to underlie major aspects of motoric, reward-seeking, and stress-coping behaviors. We sought to determine the ultrastructural substrate for this modulatory action at the level of dopaminergic perikarya in the rat ventral tegmental area (VTA). Using a dual-labeling, immunoperoxidase and immunogold-silver method, we localized antisera directed against leu ${ }^{5}$-enkephalin (ENK) and the catecholamine-synthesizing enzyme tyrosine hydroxylase (TH) in acrolein-fixed sections through the VTA. ENK-like immunoreactivity (ENKLI) was visualized within unmyelinated axons and in axon terminals. In terminals, ENK-LI was densely localized to one or more dense-cored vesicles and either densely or lightly detected surrounding small clear vesicles. Immunoreactive dense-cored vesicles were occasionally associated with the presynaptic specialization but were more frequently detected at distant sites along the plasmalemmal surface, often in apposition to astrocytic processes. ENK-immunoreactive terminals formed both symmetric and asymmetric synapses, most frequently on large proximal dendrites. Direct appositions without glial separation were also detected between terminals containing ENK-LI and other ENK-labeled or unlabeled terminals. In contrast to ENK-LI, immunolabeling for TH was primarily detected within perikarya and dendrites in the VTA. Of the ENK-immunoreactive terminals that formed synaptic contacts in single sections, approximately $50-60 \%$ were in association with TH-labeled dendrites. The remainder formed synapses on dendrites lacking detectable immunoreactivity for TH. Multiple ENK-immunoreactive terminals occasionally formed convergent synaptic contacts on single TH-labeled or unlabeled dendrites. Furthermore, individual ENK-labeled terminals sometimes formed divergent contacts on two TH-labeled or unlabeled dendrites. When a single ENK-immunoreactive terminal made dual synaptic contacts on TH-labeled dendrites, the latter were usually in close apposition to one another. These findings rep-

\footnotetext{
Received May 24, 1991; revised Sept. 24, 1991; accepted Nov. 11, 1991.

This work was supported by Public Health Service Grants NS08193 (to S.R.S.) and MH40342, MH00078, and DA04600 (to V.M.P.). We are grateful to Drs. Tong $\mathrm{H}$. Joh and Cory Abate for the generous supply of rabbit tyrosine hydroxylase antiserum.

Correspondence should be addressed to Susan R. Sesack, Division of Neurobiology, Cornell University Medical College, 411 East 69 th Street, New York, NY 10021.

Copyright (C) 1992 Society for Neuroscience $0270-6474 / 92 / 121335-16 \$ 05.00 / 0$
}

resent the first ultrastructural demonstration that opioid peptide-containing terminals provide a direct synaptic input to dopaminergic, as well as nondopaminergic, neurons in the VTA. In addition, the morphological evidence suggests that endogenous opioid peptides (1) may be released from nonsynaptic sites, (2) may modulate the release of transmitters from other terminals, and/or (3) may synchronize the activity of multiple neuronal targets in the VTA. These results provide a number of morphological substrates through which opiates may directly or indirectly regulate activity in mesocorticolimbic dopaminergic pathways.

Enkephalins or endorphins, as well as their synthetic analogs and enkephalinase inhibitors, activate the mesocorticolimbic dopaminergic pathway, causing an increase in dopamine release in the forebrain and a concomitant enhancement of locomotor and exploratory activity (Broekkamp et al., 1979; Kelley et al., 1980; Stinus et al., 1980; Kalivas and Richardson-Carlson, 1986; Kim et al., 1986). These effects occur following either systemic administration or direct injection of these substances into the ventral tegmental area (VTA), the source of dopaminergic perikarya. The VTA is also a crucial site for mediating the rewarding properties of opiate drugs, although the contribution of dopaminergic neurons to these reinforcing effects has not been conclusively established (Vaccarino et al., 1986; Latimer et al., 1987; Shippenberg and Herz, 1987; Van Ree and Ramsey, 1987; Di Chiara and Imperato, 1988; Koob and Bloom, 1988; Wise, 1989).

The cellular mechanism underlying the modulatory effects of opiates most likely involves a $\mu$-receptor-mediated increase in the activity of dopaminergic, as well as nondopaminergic, neurons in the VTA (Gysling and Wang, 1983; Kalivas et al., 1983; Latimer et al., 1987). In this regard, autoradiographic studies have shown a high density of $\mu$ (and a low density of $\delta$ ) receptors in ventral midbrain regions containing dopaminergic neurons (Mansour et al., 1987; Dilts and Kalivas, 1989, 1990). Furthermore, light microscopic immunocytochemical studies in rats and humans have shown opioid-immunoreactive fibers that overlap significantly with dopaminergic perikarya and dendrites in the VTA and contiguous cell groups in the substantia nigra (SN) (Johnson et al., 1980; Gaspar et al., 1983; Khachaturian et al., 1983b; Zamir et al., 1984; Fallon and Leslie, 1986).

Despite this compelling light microscopic evidence for overlap of opioid peptide and dopaminergic neuronal processes in the VTA, it has not yet been determined whether terminals containing opioid peptides make direct synaptic contact on do- 
paminergic neurons. Moreover, physiological studies suggest that the effects of opiates on the mesocorticolimbic dopaminergic system are mediated indirectly (Gysling and Wang, 1983; Matthews and German, 1984; Lacey ct al., 1989). In addition, autoradiographic studies indicate that $\mu$ - and $\delta$-opiate receptors are localized to nondopaminergic VTA cells (Dilts and Kalivas, $1989,1990)$. Thus, it might be expected that terminals containing endogenous opioid peptides form synapses principally on nondopaminergic neurons in the VTA.

We have investigated the relative frequencies with which opiate terminals form synapses on dopaminergic or nondopaminergic VTA neurons using dual-labeling immunocytochemistry and electron microscopy. Antisera directed principally against leu ${ }^{5}$-enkephalin $(\mathrm{ENK})$ were used in combination with antibodies raised against the catecholamine synthetic enzyme tyrosine hydroxylase (TH) (Joh et al., 1973; Bayer and Pickel, 1990). Our findings provide the first direct ultrastructural evidence that ENK-immunoreactive terminals in the VTA make monosynaptic contact with both TH-labeled and unlabeled dendrites. These results are discussed in relation to the functional evidence for primarily indirect effects of opiates on dopaminergic neuronal activity.

A preliminary report of this data has been recently published (Sesack and Pickel, 1990).

\section{Materials and Methods}

Fixation and immunocytochemistry. Six male Sprague-Dawley rats (250$300 \mathrm{gm}$ ) were anesthetized and perfused through the aorta with (1) 10 $\mathrm{ml}$ of heparin $(1000 \mathrm{U} / \mathrm{ml}$ ) in saline; (2) $50 \mathrm{ml}$ of $3.75 \%$ acrolcin (Polysciences) and $2 \%$ paraformaldehyde in $0.1 \mathrm{~m}$ phosphate buffer, $\mathrm{pH} 7.4$; and (3) $200 \mathrm{ml}$ of $2 \%$ paraformaldehyde. The brains were removed, cut into 4-5 mm coronal blocks, and fixed for an additional $30 \mathrm{~min}$ in $2 \%$ paraformaldehyde. Following sectioning at $40 \mu \mathrm{m}$ on a Vibratome, the tissue was treated with $1 \%$ sodium borohydride in $0.1 \mathrm{M}$ phosphate buffer, to improve immunocytochemical labeling (Leranth and Pickel, 1989). Tissue was then rinsed in $0.1 \mathrm{M}$ phosphate buffer, followed by $0.1 \mathrm{~m}$ Tris-buffered saline (TBS), $\mathrm{pH}$ 7.6. The sections were incubated for $1 \mathrm{hr}$ in $1 \%$ bovine serum albumin (BSA) in TBS to minimize nonspecific labeling, and then coincubated overnight in one of two primary antisera solutions: (1) 1:20,000 rabbit anti-leus -enkephalin (Incstar Inc.) and undiluted mouse monoclonal anti-TH (Boehringer-Mannheim), or (2) 1:50 mouse monoclonal anti-leus-enkephalin (Sera Labs) and 1:50,000 rabbit anti-TH (Joh et al., 1973).

The tissue was then processed using a dual immunoperoxidase and immunogold-silver labeling method (Chan et al., 1990). Briefly, sections were incubated in (1) 1:400 biotinylated goat anti-rabbit IgG (Vector Labs) for $30 \mathrm{~min}$, (2) 1:200 avidin-biotin peroxidase complex (ABC) (Hsu et al., 1981) (Vector Labs) for $30 \mathrm{~min}$, and (3) $22 \mu \mathrm{g}$ of 3,3'diaminobenzidine (Aldrich) and $10 \mu \mathrm{l}$ of $30 \% \mathrm{H}_{2} \mathrm{O}_{2}$ in $100 \mathrm{ml}$ of TBS for $6 \mathrm{~min}$. All incubations were performed at room temperature with continuous agitation, and sections were rinsed in TBS between incubations. Following peroxidase reaction, the tissue was transferred to $0.01 \mathrm{~m}$ phosphate-buffered saline (PBS), $\mathrm{pH} 7.4$; blocked in $0.8 \%$ BSA and $0.1 \%$ gelatin in PBS; and incubated for $3 \mathrm{hr}$ in 1:50 goat anti-mouse IgG, conjugated with $1 \mathrm{~nm}$ gold (AuroProbe One, Amersham). The sections were then rinsed in PBS, postfixed with $2 \%$ glutaraldehyde, rinscd again in PBS, and then rinsed in distilled water. The conjugated gold particles were enhanced by treatment with silver solution (IntenSE, Amersham). The optimal silver enhancement times for electron microscopy were determined by empirical observation: 4-5 min for $\mathrm{TH}$ and 6-7 min for enkephalin antibodies. For light microscopic detection of gold-silver labeling, the sections were reacted for an additional 3-5 min in silver solution, while monitoring the tissue with a dissecting microscope (Chan et al., 1990).

Antibody specificity. The production and specificity of TH antibodies have been previously described by Joh et al. (1973) for rabbit polyclonal antiserum and by the commercial supplier (Boehringer-Mannheim) for mouse monoclonal antibody. The specificity of mouse monoclonal and rabbit polyclonal anti-leu ${ }^{5}$-enkephalin antibodies was tested by im- munoblot analysis (Larsson, 1981). The rabbit antiserum recognized primarily leu ${ }^{5}$-enkephalin but cross-reacted significantly with met ${ }^{5}$-enkephalin and dynorphin peptides. Cross-reaction with $\beta$-endorphin was only weakly detected. The mouse monoclonal antibody produced a similar pattern of cross-reaction but was overall more specific for leu $\mathrm{u}^{5}$ enkephalin (Milner et al., 1989). Because of the described cross-reactions, the immunolabeling produced by either of these antibodies in tissue is referred to as ENK-like immunoreactivity (ENK-LI). Additional controls for specificity included replacement of primary antiserum with normal serum or antiserum preadsorbed with leu ${ }^{5}$-enkephalin.

Light and electron microscopy. Alternate serial sections for light microscopy were mounted on gelatin-coated slides, dehydrated, and coverslipped. For electron microscopy, adjacent sections were postfixed in $2 \%$ osmium tetroxide in $0.1 \mathrm{~m}$ phosphate buffer for $1 \mathrm{hr}$, dehydrated through graded ethanols and propylene oxide, and embedded in Epon 812 (Leranth and Pickel, 1989). Ulirathin sections were collected in serial order from the outer surface of the tissue, counterstained with uranyl acetate and lead citrate, and examined with Philips 201 and CM10 electron microscopes.

Quantitative ultrastructural analysis. A semiquantitative method was used to assess the relationship of terminals containing ENK-LI with $\mathrm{TH}$-labeled soma and dendrites in the VTA. The areas sampled included primarily the paranigral and parabrachial regions (Bayer and Pickel, 1990); no attempt was made to comparatively analyze different subdivisions of the VTA. Ultrathin sections were cut from the outer surface of one to five Vibratome sections per animal, and a $3250 \mu \mathrm{m}^{2}$ area (boundary of the grid mesh) of the Epon-tissue interface was examined in single sections. All ENK-immunoreactive terminals within a total area of $65,000 \mu \mathrm{m}^{2}$ were photographed at a magnification of $20,000 \times$. The morphology of terminals containing ENK-LI and their synaptic associations with $\mathrm{TH}$-immunoreactive neurons were analyzed in these micrographs.

To reduce false negatives due to inadequate penetration of antisera, only sections near the surface of the tissue were examined, and only micrographs containing both ENK-immunoreactive terminals and $\mathrm{TH}$ labeled cellular elements were analyzed. To maximize further the detection of each antigen, the more sensitive $A B C$ peroxidase method was used alternatingly to localize FNK or TH in tissue, in combination with gold-silver labeling for the other antigen. Since the number of terminals with detectable ENK-LI was considerably lower with the gold-silver method than when $\Lambda \mathrm{BC}$ peroxidase was used, the majority of sampling was done using the latter marker for ENK-LI. However, the results were pooled for quantitative estimates. With regard to gold-silver labeling for either ENK or TH localization, the number of immunogold particles within most labeled neuronal structures was at least five times higher than background (see Chan et al., 1990) and did not require further quantification. In cases of relatively sparse gold-silver particles, labeled structures were examined in two or more serial sections to determine if immunoreactivity was specific.

The classification of identified cellular elements was based on the descriptions of Peters et al. (1991). Neuronal somata were identified by the presence of a nucleus. Dendrites were identified by the presence of densities postsynaptic to axon terminals and usually contained rough endoplasmic reticulum. Dendrites were arbitrarily defined as proximal (i.c., large) if thcy wcre greater than $0.7 \mu \mathrm{m}$ in diameter. Axon terminals contained numerous synaptic vesicles and were at least $0.2 \mu \mathrm{m}$ in diameter. Asymmetric synapses were identified by thick postsynaptic densities [Gray's type I (Gray, 1959)], while symmetric synapses had thin densities (Gray's type II). Nonsynaptic contacts, or appositions, were defined as close parallel membrane associations of axon terminals or dendrites, which lacked recognizable specializations, but were otherwise not separated by astrocytic processes.

\section{Results}

\section{Light microscopy}

Peroxidase immunoreactivity for ENK was localized in punctate varicosities diffusely distributed throughout the VTA and in the adjacent medial portions of the SN (Fig. la). The distribution of ENK labeling overlapped significantly with that of TH-immunoreactive perikarya and thick processes (most likely dendrites) in adjacent sections (Fig. $1 b$ ). Immunoperoxidase and immunogold-silver markers for TH or ENK-LI were not readily distinguishable by light microscopy in the same sections, due 
to their similar brown color at shorter silver incubation times and the masking effects of the gold-silver product after longer incubations (Chan et al., 1990). However, these markers were readily differentiated by electron microscopy.

\section{Electron microscopy}

Distribution of ENK and TH immunoreactivity. Ultrathin sections for electron microscopic analysis were taken from dually labeled tissue through regions of the VTA shown in Figure 1. Within these regions, an extensive overlap of ENK-LI and TH labeling was evident (Fig. 2). Peroxidase labeling for ENK was localized primarily within unmyelinated axons or axon terminals, while gold-silver immunoreactivity for TH was localized largely within perikarya or dendrites. ENK labeling within dendrites, or TH labeling within terminals was only rarely detected (not shown). ENK-immunoreactive terminals within the same field appeared to contain different levels of peroxidase product, and could be roughly separated into two groups. The majority of ENK-immunoreactive terminals $(91 / 145,63 \%)$ were densely labeled, with reaction product heavily distributed along the outer membranes of mitochondria and small clear vesicles, and throughout the lumen of many, but not all, large dense-cored vesicles (Fig. 2). Lightly labeled terminals (54/145, 37\%) exhibited immunoperoxidase product either largely confined to densecored vesicles, or inhomogeneously distributed throughout the terminal (Fig. 2). TH-labeled perikarya and dendrites within the same field also exhibited varying densities of gold-silver particles. However, even in the lightly labeled processes, gold-silver particles were always more numerous within labeled dendrites than in comparable areas of the surrounding neuropil (Fig. 2). Furthermore, specific gold labeling could be verified in adjacent serial sections. The morphological features of TH-labeled dendrites and their synaptic input from unlabeled terminals have been previously described (Bayer and Pickel, 1990) and were not examined in further detail.

In a few experiments, the markers for dual labeling were switched, with gold-silver used to detect ENK-LI and peroxidase used to localize TH-immunoreactivity (see Fig. 7). In these cases, many fewer ENK-immunoreactive processes were encountered, and the small number of gold-silver particles in labeled terminals were often confined to large dense-cored vesicles (see Fig. 7). Thus, it was not possible to distinguish two different levels of ENK-LI with this method. Nevertheless, specific goldsilver labeling was verified in each case by examining adjacent serial sections. While the bulk of quantitative analysis that fol- lows was based on peroxidase labeling for ENK-LI, 47 ENKimmunoreactive terminals labeled with the gold-silver method were included.

ENK-immunoreactive terminals. Terminals containing ENKLI (labeled either by peroxidase or gold-silver methods) were small to large. The shortest diameter ranged from 0.2 to $1.5 \mu \mathrm{m}$, with a mean diameter of $0.54 \pm 0.21 \mu \mathrm{m}$ (mean $\pm \mathrm{SD} ; n=$ 192). ENK-immunoreactive terminals contained occasional mitochondria, numerous small clear vesicles, and often one or more large dense-cored vesicles. The content of dense-cored vesicles in single sections through each terminal varied from 0 to 11 , with a mean of $1.64 \pm 1.89(n=192)$. The punctate peroxidase labeling over immunoreactive dense-cored vesicles was exclusively seen near the surface of sections exposed to ENK antisera and was substantially more electron dense than the core of nonimmunoreactive dense-cored vesicles within the surrounding neuropil (Fig. 2, inset).

Labeled dense-cored vesicles ranged in size from 60 to 120 $\mathrm{nm}$ and appeared larger than vesicles lacking detectable peroxidase immunoreactivity (Figs. $2,3 a$ ). This difference may at least partly reflect accumulation of peroxidase product. However, a small, but statistically significant difference in size was also noted between immunoreactive and nonimmunoreactive dense-cored vesicles in tissue labeled with immunogold-silver for ENK (see Fig. 7). ENK-labeled dense-cored vesicles had a mean diameter of $85.78 \pm 13.98 \mathrm{~nm}$ (mean $\pm \mathrm{SD} ; n=90$ ), while unlabeled dense-cored vesicles were $81.42 \pm 13.94 \mathrm{~nm}$ in diameter $(n=113)(p<0.05$, independent $t$ test $)$.

Terminals containing ENK-LI formed either symmetric or asymmetric synaptic junctions in approximately equivalent proportions (Table 1). Symmetric synapses had thin pre- and postsynaptic densities and were often short and punctate (see Figs. $3 a, 5,6)$. Asymmetric junctions were characterized by thick postsynaptic densities, usually associated with less prominent presynaptic thickenings (Fig. 4). However, ENK-immunoreactive terminals sometimes also formed junctions possessing both pre- and postsynaptic thickenings (Fig. $3 b$ ). These were classified as asymmetric on the basis of the postsynaptic density. In addition, junctions with thick densities, but for which the pre- and postsynaptic membranes were not readily apparent due to an oblique plane of section or dense immunoreactivity (see Fig. 7), were also included in this category. Some of these junctions were verified as asymmetric (i.e., having thick postsynaptic densities) when viewed using a goniometer stage on a Philips CM10 electron microscope. ENK-labeled terminals forming asym-

\footnotetext{
Figure 1. Light micrographs illustrating the overlapping distribution of peroxidase immunoreactivity for ENK $(a)$ or TH $(b)$ in the VTA. $a$, ENKLI is localized throughout the ventral midbrain and substantia nigra, including the medial zona compacta $\left(S N_{c}\right)$ and zona reticulata $\left(S N_{r}\right)$. At higher magnification (inset) using Nomarski optics, reaction product can be seen within punctate processes (large arrow) surrounding unlabeled perikarya (small arrows). $b$, Immunoreactivity for TH is also intensely localized throughout the VTA and medial $S N_{c}$ but is much less dense within the $S N_{r}$. At higher magnification (inset), TH immunoreactivity is evident within pcrikarya and thick processes having the appearance of dendrites (arrow). The boxed regions, including parts of the paranigral and parabrachial subdivisions of the VTA, correspond to the approximate areas sectioned for ultrastructural analysis. $m l$, medial lemniscus; $m p$, mammillary peduncle (near the ventral brain surface). Scale bar, $600 \mu \mathrm{m}$ for low magnification and $120 \mu \mathrm{m}$ for insets.

Figure 2. Low-power electron micrograph demonstrating an abundance of axon terminals containing immunoperoxidase for ENK-LI (ENK-T) and dendrites containing immunogold-silver labeling for TH $(T H-D)$ in the VTA. $E N K-T$ s are either densely labeled $\left(E N K-T_{D}\right)$ and exhibit peroxidase product surrounding small clear vesicles and filling large dense-cored vesicles (small arrows) or lightly labeled $\left(E N K-T_{L}\right)$, with reaction product inhomogeneously distributed to some small clear vesicles or largely restricted to dense-cored vesicles. Unlabeled terminals $(u T)$ contain neither peroxidase product nor gold-silver labeling. The difference in electron density between labeled (small arrows) and unlabeled (arrowheads) dense-cored vesicles is shown more clearly at higher magnification in the inset (corresponding to the boxed region). Immunogold-silver labeling for TH is seen as dense, punctate, or irregularly shaped deposits selectively localized to dendrites. Gold-silver labeling is absent from unlabeled dendrites $(u D)$ and is rarely detected within the surrounding neuropil. In some cases, ENK-Ts can be seen in synaptic contact (long arrows) with TH-Ds. Scale bars, $0.5 \mu \mathrm{m}$ in the full micrograph and $0.25 \mu \mathrm{m}$ in the inset.
} 

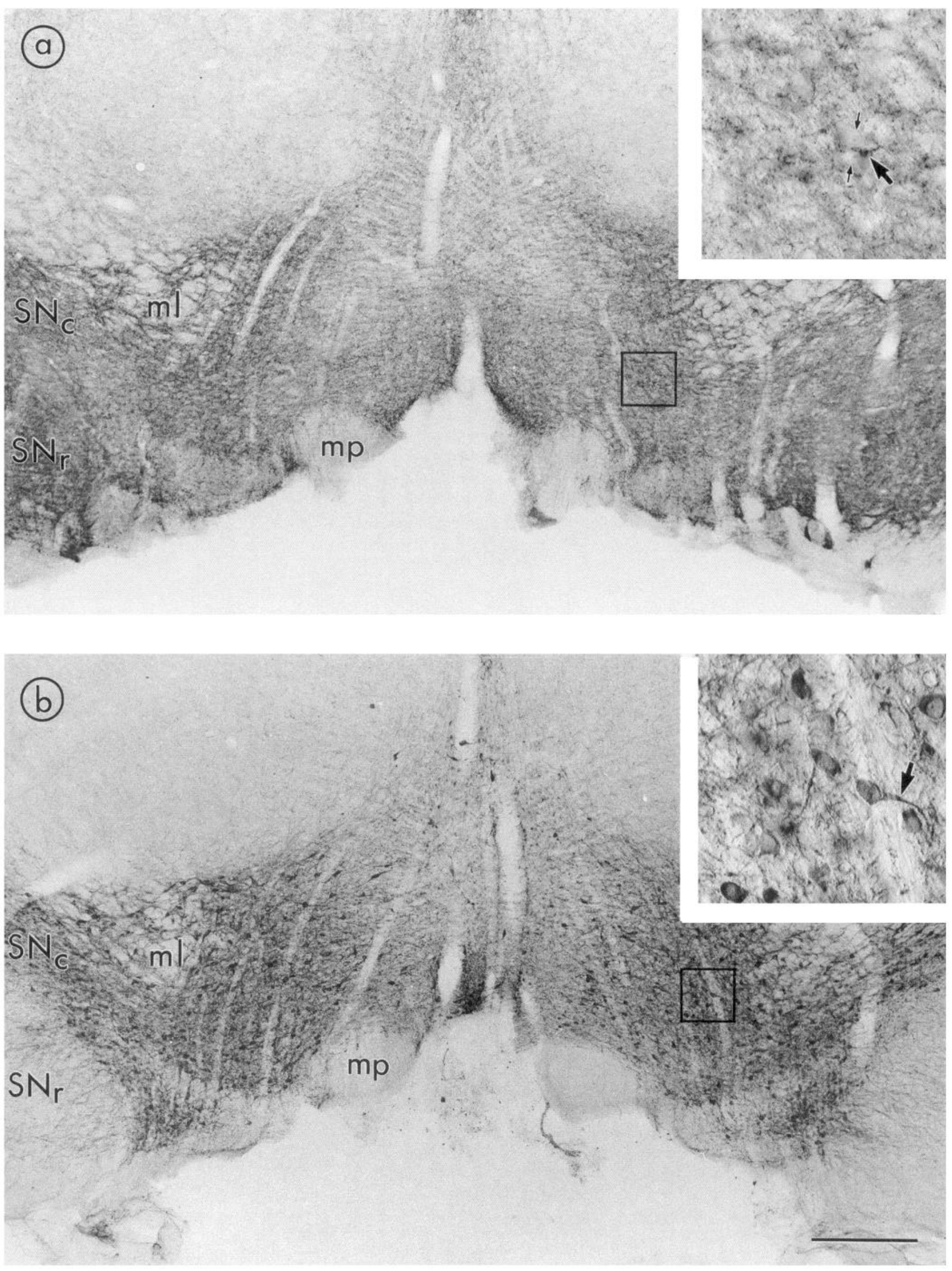


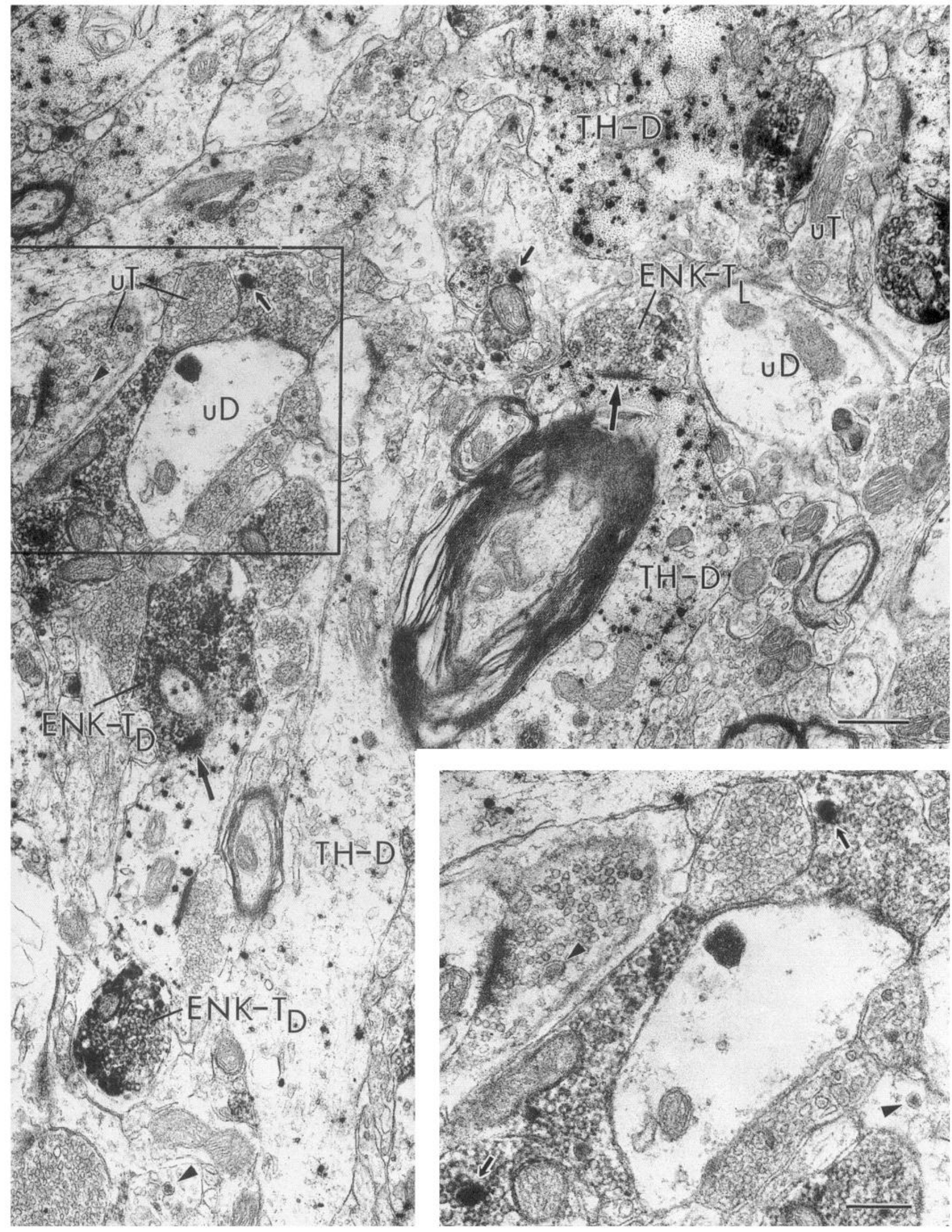


Table 1. Morphological and synaptic features of terminals containing ENK-LI in the rat VTA

\begin{tabular}{lcc} 
& \multicolumn{2}{c}{ Type of synaptic association } \\
\cline { 2 - 3 } & Symmetric & Asymmetric \\
\hline Total number and percentage & $62 / 121$ & $59 / 121$ \\
$\quad$ of total & $51 \%$ & $49 \%$ \\
Cross-sectional diameter & $0.52 \pm 0.18$ & $0.60 \pm 0.23^{*}$ \\
$\quad(\mu \mathrm{m}$, mean $\pm \mathrm{SD})$ & & \\
Content of dense-cored & $1.60 \pm 1.69$ & $1.63 \pm 1.76$ \\
vesicles (mean \pm SD) & & \\
Presynaptic localization of & $11 / 44^{* *}$ & $2 / 41$ \\
dense-cored vesicles & $(25 \%)$ & $(5 \%)$ \\
Junction with all dendrites & $42 / 62$ & $40 / 59$ \\
$\geq 0.7 \mu \mathrm{m}$ diameter & $(68 \%)$ & $(68 \%)$ \\
Axonal apposition & $25 / 62$ & $18 / 59$ \\
& $(40 \%)$ & $(30 \%)$ \\
Astrocytic apposition & $40 / 62$ & $39 / 59$ \\
& $(65 \%)$ & $(66 \%)$ \\
Junction with TH-labeled & $28 / 62$ & $30 / 59$ \\
dendrite & $(45 \%)$ & $(51 \%)$ \\
\hline
\end{tabular}

Numbers are based on analysis of at least $3250 \mu \mathrm{m}^{2}$ area of one to five vibratome sections from each of six animals (total area of $65,000 \mu \mathrm{m}^{2}$ ).

${ }^{*}, p<0.05$, independent $t$ test; ${ }^{* *}, p<0.05, \chi^{2}$ test.

metric synapses were significantly larger than those making symmetric junctions. However, with regard to other morphological features, there was little difference between these terminal types (Table 1). Terminals containing ENK-LI formed symmetric or asymmetric synapses primarily on proximal (i.e., large) dendrites (Table 1) and less frequently on distal dendritic processes, including spines. ENK-immunoreactive terminals in contact with neuronal perikarya were not detected.

Of 85 ENK-labeled terminals that formed synaptic junctions and contained one or more immunoreactive dense-cored vesicles in single sections, $72(85 \%)$ exhibited dense-cored vesicles localized to sites away from the synaptic contact zone, either within the center of the terminal or along the plasmalemmal surface (Figs. 2, 3, 5b, 6, 7). Within the remaining 13 ENKimmunoreactive terminals $(15 \%)$, at least one of the dense-cored vesicles was localized in close proximity to the presynaptic specialization (Figs. $3 a, 6,7$ ), and the majority of these formed symmetric type synapses (Table 1). This difference in synaptic localization of dense-cored vesicles within symmetric versus asymmetric terminals was significant by $\chi^{2}$ analysis (Table 1 ).

ENK-labeled terminals were sometimes apposed to dendrites (Figs. $3 b, 6$ ) or other terminals (Fig. $5 a$ ), without forming recognizable synaptic specializations in single sections. In the case of appositions to dendrites, such close proximity was sometimes associated with synapse formation along adjacent portions of the plasmalemma, which could be observed in serial sections (Fig. 6). Axoaxonal appositions were detected either between ENK-immunoreactive and unlabeled terminals $(62 / 78,80 \%)$, or between two processes containing ENK-LI (16/78, 20\%) (Fig. $5 a)$. The axonal appositions were classified as "presynaptic" $(60 / 78,77 \%)$ when one of the apposed terminals made synaptic contact with a dendritic process (Fig. $5 a$ ), and as "axoaxonal" $(18 / 78,23 \%)$ when neither terminal formed a synaptic contact with a dendrite in the section examined. The remaining ENK-

Figure 3. Electron micrographs depicting peroxidase ENK-LI within terminals $(E N K-T)$ in synaptic contact with unlabeled dendrites $(a)$ or a dendrite containing gold-silver labeling for TH $(b)$. a, ENK-Ts form symmetric synapses (long arrows) on small (left) or large (right) unlabeled dendrites $(u D)$. Both terminals are of the type containing dense peroxidase product $\left(E N K-T_{D}\right)$ rimming small clear vesicles, as well as filling densecored vesicles (small arrows). The labeled dense-cored vesicle in the terminal on the left (small arrow) is in close proximity to the presynaptic specialization. Within an adjacent section (inset), the symmetric synapse can be seen more clearly (long arrow), while a different dense-cored vesicle appears along a nonsynaptic portion of the plasmalemma (short arrow). In the terminal on the right, the labeled dense-cored vesicle (small arrow) is also distal to the synaptic junction (long arrow) and is near the plasmalemmal surface in apposition to an astrocytic process $(*)$. For comparison, arrowheads indicate unlabeled dense-cored vesicles, one within the left $E N K-T_{D}$ and the other within an unlabeled terminal $(u T)$. An astrocytic process surrounds the $E N K-T_{D}$ and its dendritic target on the left, and separates the $E N K-T_{D}$ from an adjacent process (most likely a dendrite) containing gold-silver labeling for TH $(T H-D)$. $b$, An ENK-T of the lightly labeled type $\left(E N K-T_{L}\right)$ contains peroxidase product exclusively within a dense-cored vesicle (small arrow) and forms a synapse (curved arrow) with a proximal dendrite containing gold-silver reaction product for TH $(T H-D)$. Both the pre- and postsynaptic plasmalemmal surfaces exhibit membrane thickenings. However, the aggregates of small clear vesicles (scv) presynaptically suggest that this is a synapse rather than a punctum adherens. The $E N K-T_{L}$ is also in close apposition (open arrow) to a $u D$, without forming a synapse. The labeled dense-cored vesicle (small arrow) is locatcd away from the synaptic contact zonc, near a plasmalemmal surfacc apposed to an astrocytic process (*). An unlabeled dense-cored vesicle (arrowhead) is also visible within the $E N K-T_{L}$, while a different ENK-labeled dense-cored vesicle (small arrow) can be seen within a small process adjacent to the TH-D. Scale bar, $0.25 \mu \mathrm{m}$.

Figure 4. Electron micrographs illustrating intense ENK-immunoreactive terminals $\left(E N K-T_{D}\right)$ forming asymmetric synapses on TH-labeled $(a$ and $c$ ) and unlabeled $(b)$ dendrites. $a$, A densely labeled ENK-T $\left(E N K-T_{D}\right)$ forms an asymmetric synapse (curved arrow) on a large TH-labeled dendrite $(T H-D)$, identified by gold-silver deposits. The target dendrite also receives synaptic input from an unlabeled terminal (short arrow) and is in close apposition to another TH-D (facing arrowheads). The $E N K-T_{D}$ is apposed along one surface by an astrocytic process $\left({ }^{*}\right) . b$, An ENK-T containing dense peroxidase product $\left(E N K-T_{D}\right)$ forms an asymmetric synapse associated with a postjunctional dense body (curved arrow) on a proximal dendrite. This dendrite lacks significant gold-silver deposits greater than hackground (one grain is circled), and is therefore considered unlabeled $(u D)$. Several unlabeled terminals $(u T \mathrm{~s})$ are in direct contact with the $u D$; one of these makes a clearly defined asymmetric junction $(s h o r t$ arrow). One surface of the $E N K-T_{D}$ is apposed to an astrocytic process $\left(^{*}\right)$. An adjacent $T H-D$ receives synaptic contact (short arrow) from a $u T$. $c$, A spine lacking gold-silver particles arises from a proximal $T H-D$ and receives an asymmetric synapse (curved arrow) from an ENK-T. This ENK-T is probably of the densely labeled type $\left(E N K-T_{D}\right)$, since ENK-LI is readily detectable, despite the absence of observable dense-cored vesicles. The $T H-D$ is in close apposition to another $T H-D$ (facing arrowheads) and also receives input from a $u T$ (short arrow). Scale bar, $0.25 \mu \mathrm{m}$.

Figure 5. Electron micrographs demonstrating divergent and convergent synaptic contacts formed by terminals containing ENK-LI $(E N K-T)$ on TH-labeled and unlabeled dendrites. $a$, A single ENK-T containing dense immunoreactivity $\left(E N K-T_{D}\right)$ forms symmetric synapses $(l o n g$ arrows $)$ on two different dendrites, which are also contacted by unlabeled terminals $(u T)$ (short arrows). The larger dendrite contains sparse gold-silver particles (circled) indicating immunoreactivity for TH $(T H-D)$, while the smaller dendrite has only one gold-silver particle (circled) and is considered unlabeled $(u D)$. The density of gold-silver labeling in the large $T H-D$ appears qualitatively less than in other $T H-D$ s in the same field (left side of micrograph), or in neighboring sections (as seen in $b$ ). However, the specificity of immunoreactivity was confirmed by analysis of adjacent serial sections. The large $E N K-T_{D}$ shows appositional contacts (facing arrowheads) with a smaller, though also intensely labeled ENK-T (large arrow), and a $u T$. $b$, ENK-Ts containing light peroxidase labeling $\left(E N K-T_{t}\right)$ and immunoreactive dense-cored vesicles (small arrows) form convergent symmetric synapses (long arrows) on a common dendrite labeled with gold-silver immunoreaction product for TH $(T H-D)$. One of the ENK-Ts is surrounded on its nonsynaptic plasmalemmal surface by an astrocytic process $(*)$. Scale bars, $0.5 \mu \mathrm{m}$. 

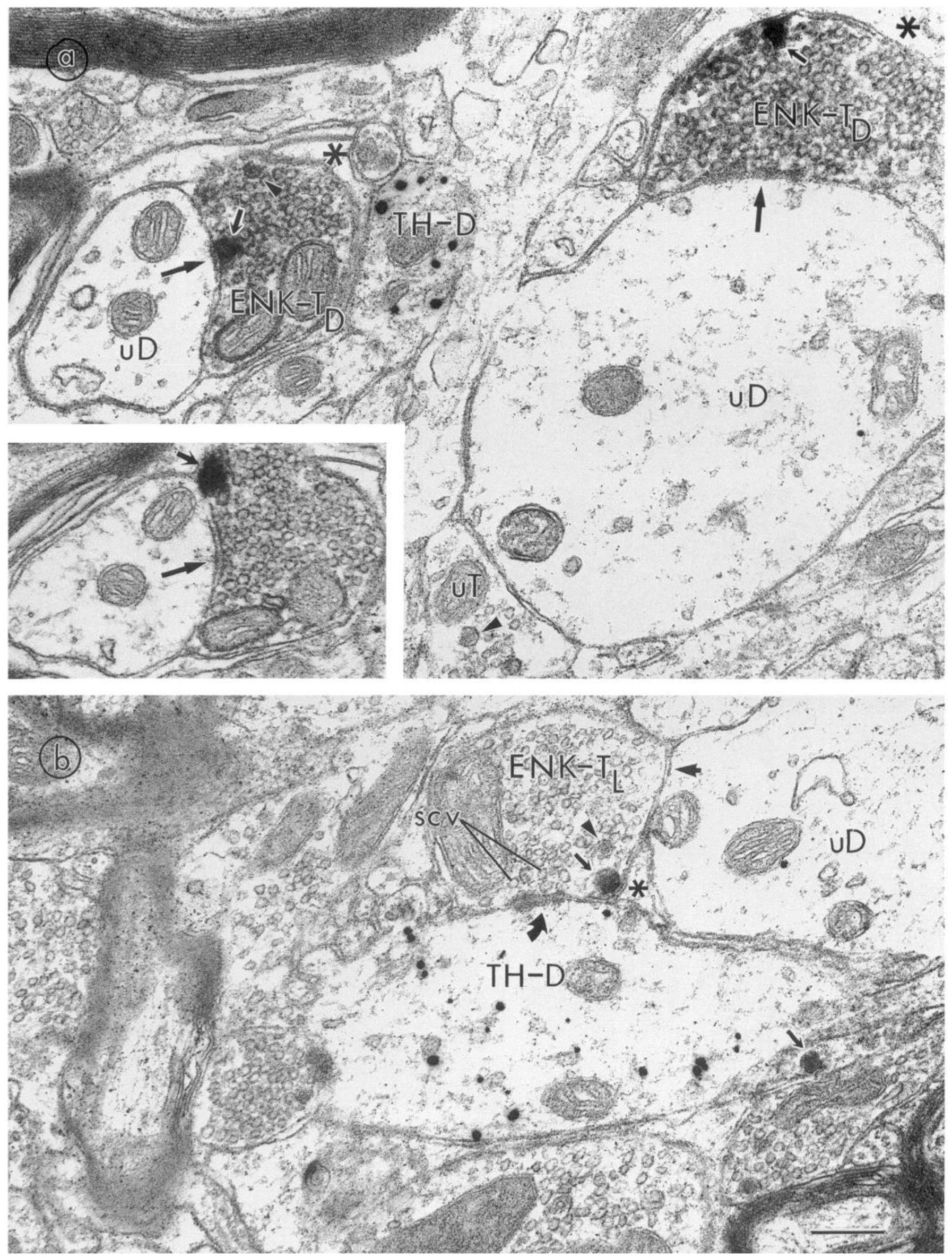

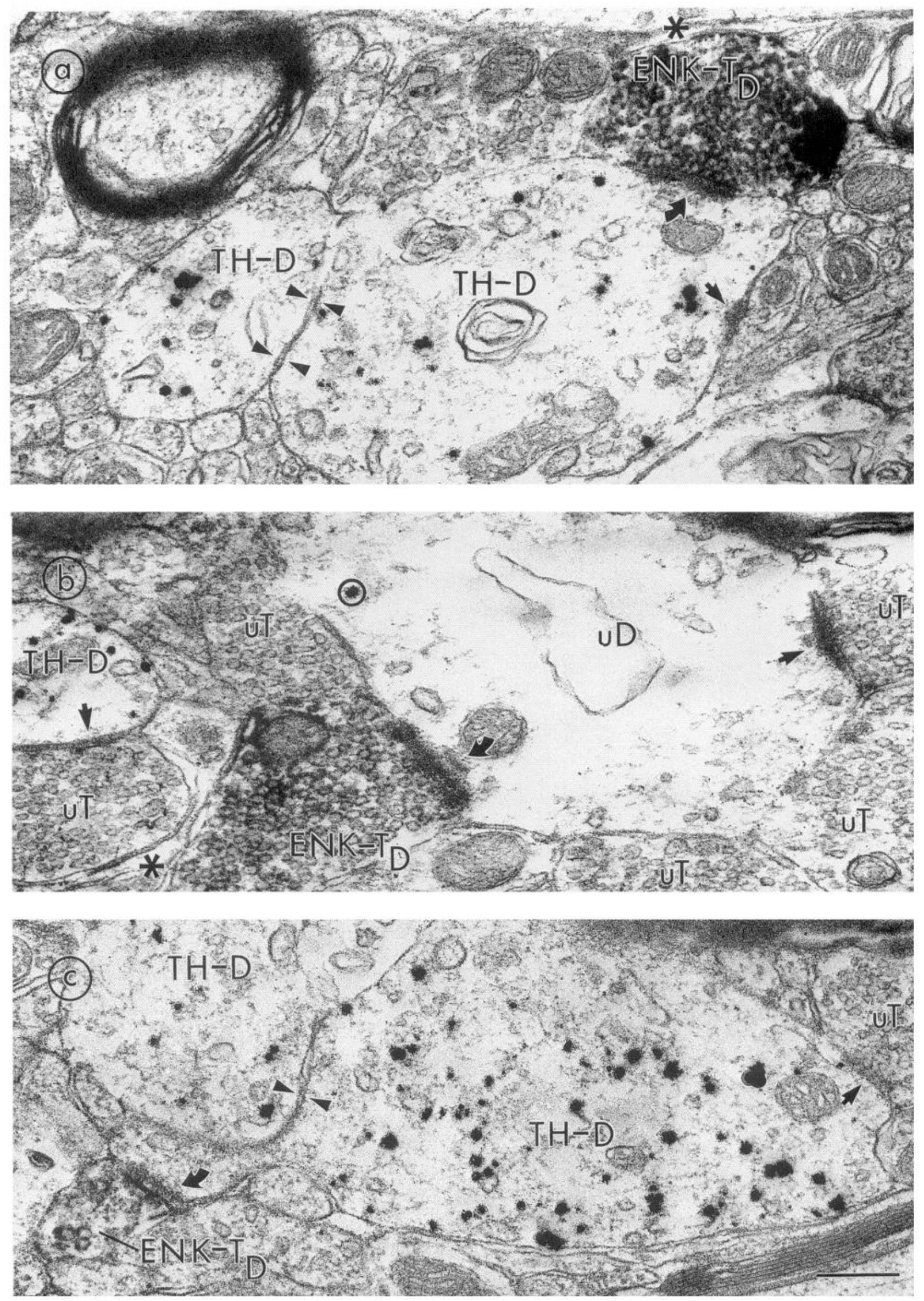

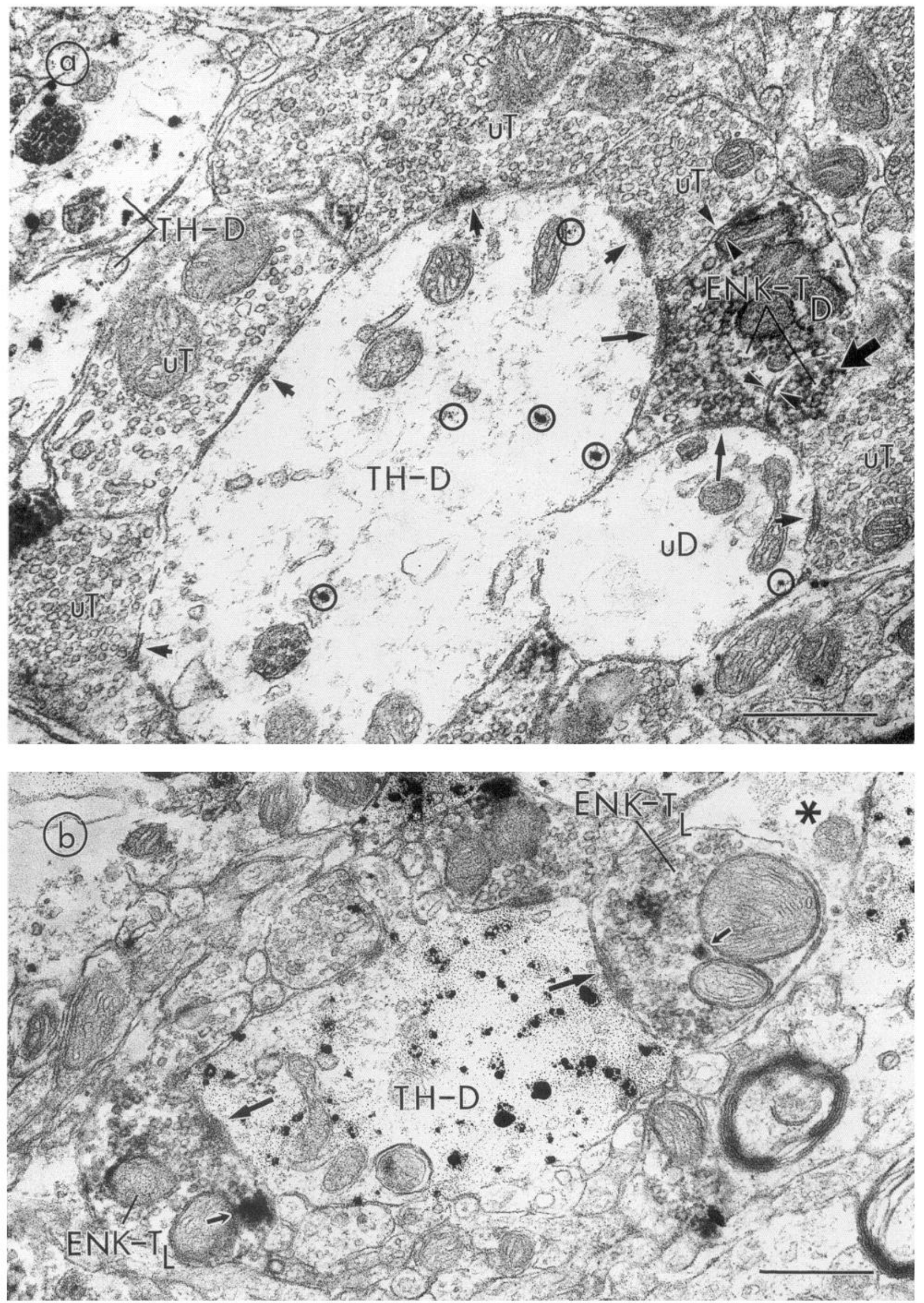
Table 2. Relationship ${ }^{a}$ of ENK-immunoreactive terminals to THlabeled and unlabeled dendrites in the rat VTA

Type of association

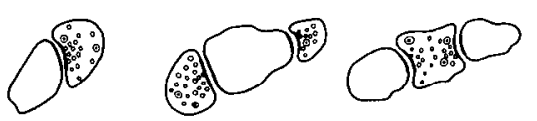

Single ENK Two ENK Single ENK terminal on terminals on terminal on single single two

Dendritic relationship dendrite dendrite dendrites

TH-labeled dendrites

$(n=83)$ $53(35 \%)$

$12(8 \%)$

$18(12 \%)^{b}$

Unlabeled dendrites

$(n=69)$

Total number of

dendrites $(n=152)$

$57(38 \%)$

$8(5 \%)$

$4(3 \%)$

$110(73 \%) \quad 20(13 \%)$

$22(15 \%)$

Numbers based on analysis of at least $3250 \mu \mathrm{m}^{2}$ area of one to five vibratome sections from each of six animals (total area of $65,000 \mu \mathrm{m}^{2}$ ).

- Includes all synapses and appositions not separatcd by glia.

${ }^{b}$ Includes inputs to two TH-labeled dendrites, or one TH-labeled and one unlabeled dendrite.

immunoreactive terminals in the analyzed micrographs (24/ 192) exhibited no synaptic or appositional contacts with other neuronal processes in single sections. The majority of ENKlabeled terminals $(115 / 192,60 \%)$ had at least one surface in apposition to an astrocytic process (Figs. 2-7); often, immunoreactive dense-cored vesicles were localized at these sites (Figs. $3,6)$. Wrapping of both pre- and postsynaptic elements of ENKlabeled synapses by astrocytes was frequently observed (Fig. $3 a$ ). ENK-immunoreactive tcrminals forming symmctric or asymmetric synapses did not differ with respect to the frequency of axonal appositions or glial associations (Table 1).

Relationship between ENK- and TH-immunoreactive processes. Terminals containing ENK-LI frequently formed synaptic contacts on large and small TH-labeled dendrites in the VTA (Figs. $2 ; 3 b ; 4 a, c ; 5-7$ ). Three different types of synaptic arrangement involving ENK-labeled terminals and TH-labeled or unlabeled dendrites were defined in single sections (Table 2). (1) The simplest type involved a single ENK-labeled terminal contacting a single TH-labeled or unlabeled dendrite (Figs. 3, 4). Many times these dendrites received convergent input from unlabeled terminals making either symmetric $(55 / 84,65 \%)$ or asymmetric (29/84, 35\%) synapses. (2) ENK-labeled terminals sometimes converged on common dendrites with other terminals containing ENK-LI (Fig. 5b). (3) Finally, individual ENKimmunoreactive terminals formed divergent contacts with two TH-labeled dendrites (Figs. 6, 7), one TH-labeled and one unlabeled dendrite (Fig. 5a), or two unlabeled dendrites (not shown). In one case, an ENK-labeled dense-cored vesicle was localized to the presynaptic specialization adjacent to one dendrite, while small clear vesicles were aggregated presynaptically at the junction with the second dendrite (Fig. 6). Furthermore, the two dendrites contacted by a single ENK-immunoreactive terminal were sometimes in close apposition to each other (Fig. 6). Overall, ENK-immunoreactive terminals contacting TH-labeled versus unlabeled dendrites did not differ significantly in density of ENK-LI, size, synaptic type (symmetric vs. asymmetric; Table 1), content of dense-cored vesicles, or frequency of glial contacts.

\section{Discussion}

Using dual-labeling peroxidase and gold-silver immunocytochemical methods, we determined that terminals containing immunorcactivity for ENK-like peptide form monosynaptic contact with TH-labeled and unlabeled dendrites in the rat VTA. Multiple ENK-immunoreactive terminals sometimes provided convergent inputs to single TH-labeled or unlabeled dendrites, while individual ENK-labeled terminals occasionally formed divergent contacts with two or more dendrites (TH labeled or unlabeled). These results suggest that terminals containing opioid peptides are uniquely positioned to provide a significant and direct, though potentially complex, input to dopaminergic and nondopaminergic neurons in the VTA.

\section{Methodological considerations}

\section{Dual immunoperoxidase and immunogold-silver labeling}

The findings of the present study are based largely on the use of a recently developed preembedding dual immunocytochemical method, which combines peroxidase with gold-silver markers (Chan et al., 1990). The use of $1 \mathrm{~nm}$ gold particles provides better tissue penetration than larger gold conjugates, and the resultant immunolabeling possesses a lower background than afforded by postembedding methods (Leranth and Pickel, 1989; Chan et al., 1990). Thus, the number of silver-enhanced gold particles within specifically labeled structures was consistently higher (by at least five times) than in the surrounding neuropil and did not require further quantitative comparison. Furthermore, the detection of spatially distinct gold-silver particles within adjacent serial sections provided additional confirmation for the specificity of immunoreactivity. However, the immunogold method proved to be less sensitive than $\mathrm{ABC}$ peroxidase staining, particularly when used to detect ENK-LI within terminals in the VTA. Also, although TH-immunoreactive (presumably dopaminergic) processes were abundantly detected with this method, it cannot be ruled out that the total numbers of dopaminergic perikarya and dendrites were underestimated using the gold-silver technique. This is especially true for distal dendritic processes and spines, which sometimes lacked goldsilver particles, despite their being clearly visible in the parent proximal dendrites.

With regard to peroxidase immunoreactivity for ENK, the observed variations in intensity of labeling may reflect differences in depth from the surface of the tissue and the attendant limitations in antisera penetration. However, adjacent terminals within the same field frequently contained different levels of ENK-LI. Furthermore, light ENK immunoreactivity was sometimes detected in terminals immediately at the tissue-Epon interface, the region having the most complete access to all immunoreagents. This situation is analogous to that described for TH immunoreactivity in the VTA, which often exhibits different intensities of labeling within adjacent perikarya or dendrites, even at the surface of the tissue (Bayer and Pickel, 1990). In the case of dopaminergic neurons, this difference is likely to reflect different levels of the TH enzyme. However, in terminals containing ENK-LI, variations in intensity of immunoreactivity might reflect not only different levels, but also different types of opiod peptides (discussed below).

\section{Antibody specificity}

One limitation of the presently employed methodology relates to the cross-reactivity of the anti-leu ${ }^{5}$-enkephalin antisera. The 


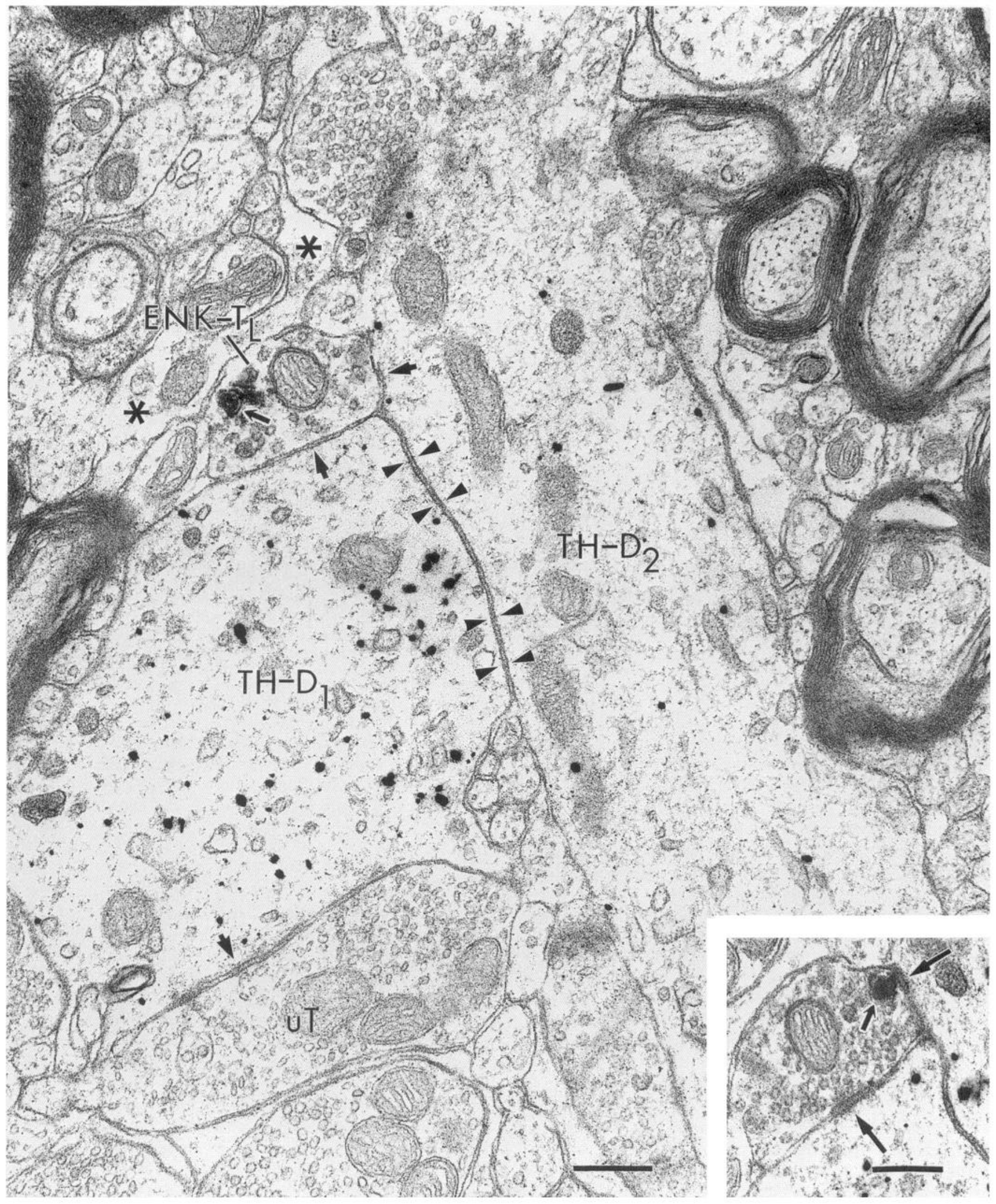

Figure 6. Electron micrograph depicting divergent synaptic input from a single terminal containing ENK-LI $(E N K$ - $T)$ to two large TH-labeled dendrites $(T H-D)$. An ENK-T contains light peroxidase labeling $\left(E N K-T_{L}\right)$ primarily within dense-cored vesicles $(s m a l l$ arrows; see also inset $)$ and is in close apposition (open arrows) to two different TH-Ds. Within an adjacent section (inset), synaptic specializations of the symmetric type (long arrows) are visible between the ENK-T and the TH-Ds. One ENK-labeled dense-cored vesicle is located at the presynaptic specialization in contact with $\mathrm{TH}-\mathrm{D}_{2}$ (inset), and the other is near the plasmalemmal surface apposed to an astrocyte $\left({ }^{*}\right)$. Aggregates of small clear vesicles are seen near the presynaptic specialization in contact with $T H$ - $D_{l}$ (inset). The TH-Ds are in close apposition to one another (facing arrowheads). TH-D, contains numerous gold-silver particles and receives a convergent input from an unlabeled terminal ( $u T$, open arrow), while $T H-D_{2}$ exhibits sparse labeling. Scale bars: full micrograph, $0.25 \mu \mathrm{m}$; inset, $0.15 \mu \mathrm{m}$. 


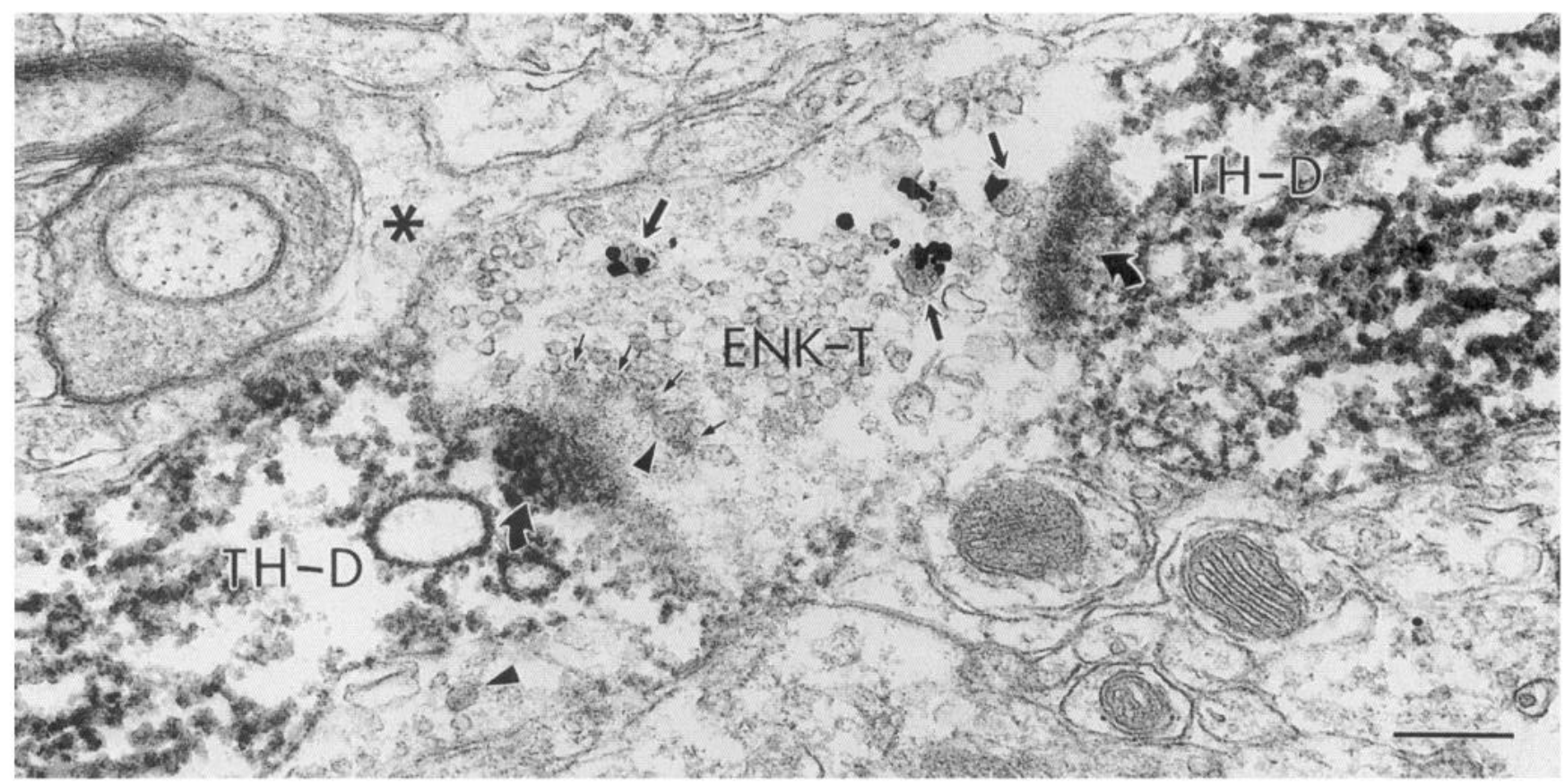

Figure 7. Electron micrograph illustrating a terminal containing gold-silver particles for ENK-LI $(E N K-T)$ in synaptic contact with peroxidasecontaining TH-labeled dendrites $(T H-D)$. An ENK-T containing several large dense-cored vesicles labeled with gold-silver particles (larger arrows) makes synaptic contact (curved arrows) with large dendrites containing peroxidase product for TH. The junctions are associated with dense thickenings (subsynaptic dense bodies at the contact on the left are indicated by the smaller arrows), although the oblique plane of section and dense peroxidase labeling preclude further characterization of pre- and postsynaptic specializations. One immunoreactive dense-cored vesicle is located near the presynaptic specialization on the right (upper large arrow), while an unlabeled dense-cored vesicle (arrowhead) is adjacent to the synaptic contact zone on the left. The other labeled dense-cored vesicles are located away from the synaptic specializations, and one nonimmunoreactive densecored vesicle is visible within an unlabeled terminal (arrowhead). The ENK-T has one surface in apposition to an astrocytic process (*). Scale bar, $0.25 \mu \mathrm{m}$.

recognition by these antisera of both leus- and mets-enkephalin in the VTA is likely, given the high levels of both peptides reported in this region (Zamir et al., 1985). However, our preliminary data using an antiserum with nearly complete specificity for dynorphin peptides (Pickel et al., 1991) suggest that the immunolabeling in the present study does not represent dynorphin-like peptides. First, the immunoreactivity produced by anti-dynorphin antiserum was considerably less dense (by light and electron microscopy) in the VTA than in the $\mathrm{SN}$, while ENK-LI was densely localized throughout both regions. Second, the detection of immunoreactivity for dynorphin antiserum required the aid of detergent to enhance penetration, while such treatment was not necessary for immunolabeling with anti-leusenkephalin antisera. Thus, we feel that the presently described ENK-LI in the VTA primarily reflects the presence of enkephalin peptides.

\section{Subcellular localization of ENK-LI}

The detection of peroxidase immunoreactivity for ENK in large vesicles that were already electron dense by virtue of osmium tetroxide and heavy metal counterstaining required careful comparison with other, apparently unlabeled dense-cored vesicles in the surrounding neuropil. Those vesicles considered to be specifically labeled were exclusively seen near the surface of sections incubated with ENK antiserum. At greater depths from the surface of these immunolabeled sections and throughout sections processed in the absence of ENK antiserum, vesicles with comparable peroxidase labeling were not seen. Furthermore, localization of ENK-LI principally to dense-cored vesicles was confirmed with the nondiffusible immunogold-silver mark- er. Finally, the detection of ENK-LI within dense-cored vesicles agrees with previous descriptions of the subcellular localization of this peptide (Pelletier and Leclerc, 1979; Pickel et al., 1980; Klein et al., 1982; Sumal et al., 1982; Inagaki et al., 1986; Milner et al., 1989; Dores et al., 1990).

\section{ENK-immunoreactive terminals}

ENK localization to dense-cored vesicles

The morphological features of axons and terminals containing ENK-LI in the VTA generally match previous reports of ENKlabeled processes in the CNS and PNS of rats and nonhuman primates (Pickel et al., 1980; Di Figlia et al., 1982; Somogyi et al., 1982; Sumal et al., 1982; Inagaki et al., 1986; Milner et al., 1989). The present finding that ENK-LI was consistently localized to dense-cored vesicles that were somewhat larger than nonimmunoreactive dense-cored vesicles suggests that opioid peptides are specifically contained within the class of "large" (i.e., greater than $70 \mathrm{~nm}$ ) vesicles associated with peptide storage (Klein et al., 1982; Dores et al., 1990; Thureson-Klein and Klein, 1990). Conversely, unlabeled dense-cored vesicles more frequently included a smaller class $(40-55 \mathrm{~nm})$, such as those associated with monoamine sequestration (Thureson-Klein and Klein, 1990).

In other systems, large dense-cored vesicles have been shown to undergo nonsynaptic exocytotic release (Zhu et al., 1986; Cuadras, 1989; reviewed by Thureson-Klein and Klein, 1990), suggesting the possibility that opioid peptides may be released nonsynaptically in the VTA. This hypothesis is supported by our observations that the majority of ENK-immunoreactive dense-cored vesicles were localized to plasmalemmal surfaces 
away from the synaptic contact zone, often in close apposition to astrocytic processes. Although some ENK-immunoreactive dense-cored vesicles were localized adjacent to the presynaptic specialization, dense-cored vesicles in other systems have been shown to lack at least one of the synaptic proteins required for conventional release mechanisms (Navone et al., 1984). Thus, definitive conclusions regarding potential sites or mechanisms of opioid peptide release in the VTA will require additional experimental evidence.

\section{Synaptic specializations}

The finding that ENK-labeled terminals in the VTA formed either symmetric or asymmetric synapses may reflect their content of different opioid peptides. ENK-immunoreactive terminals forming asymmetric synapses have been detected only rarely with anti-leu ${ }^{5}$-enkephalin antiserum (Di Figlia et al., 1982; Somogyi et al., 1982; Milner et al., 1989). However, the present findings may be partly explaincd by antiscrum cross-reaction with met $^{5}$-enkephalin, which has more frequently been localized to terminals forming asymmetric junctions (Pickel et al., 1980; Sumal et al., 1982; Inagaki et al., 1986).

It is also possible that ENK-immunoreactive terminals forming either symmetric or asymmetric synapses originate from different sources. Many of the features of ENK-immunoreactive axons and terminals in the VTA match those described for striatal collaterals (Pickel et al., 1980; Di Figlia et al., 1982; Somogyi et al., 1982), suggesting that they originate from this region (Zamir et al., 1984). However, recent studies indicate that striatal enkephalin-containing neurons project preferentially to the globus pallidus, and less frequently to the SN/VTA (Gerfen and Young, 1988; Reiner and Anderson, 1990). Thus, ENK-immunoreactive terminals in the VTA may also originate from nonstriatal sources, most notably the frontal cortex, hypothalamus, amygdala, raphe nuclei, or intrinsic VTA cells (Phillipson et al., 1979; Khachaturian et al., 1983a,b; Fallon and Leslie, 1986; Harlan et al., 1987).

Finally, ENK-labeled terminals having different synaptic specializations may contain different classical transmitters, which are colocalized with opioid peptides and released synaptically from small clear vesicles (Hökfelt et al., 1987; Thureson-Klein and Klein, 1990). For example, GABA coexists with enkephalin in striatal neurons (Reiner and Anderson, 1990), suggesting that some of the ENK-immunoreactive terminals in the VTA may colocalize this inhibitory amino acid transmitter. In this regard, the demonstration that multiple ENK-immunoreactive terminals made synaptic contacts (of symmetric type) onto single dendrites is remarkably similar to the results recently reported for GABAergic terminals in the VTA (Bayer and Pickel, 1991). Furthermore, we have obtained preliminary evidence that a population of VTA terminals does indeed contain both opioid peptides and GABA (S. R. Sesack and V. M. Pickel, unpublished observations).

In classical transmitter systems, a correlation has been made between synaptic morphology and physiological function. For example, nerve terminals containing inhibitory transmitters form primarily symmetric synapses, while terminals containing excitatory transmitters form mostly asymmetric synaptic junctions (Carlin et al., 1980; Peters et al., 1991). However, for putative peptide transmitters, the correlation between morphology and physiology may not be so tenable. For example, opioid peptide-containing nerve terminals form both symmetric and asymmetric synapses (Pickel et al., 1980; Di Figlia et al., 1982; Somogyi et al., 1982; Sumal et al., 1982; Inagaki et al., 1986; Milner et al., 1989; present study), whilc opiates primarily mediate an inhibitory physiological action (Nicoll et al., 1977; Morita and North, 1981; Williams et al., 1982). In a wellknown exception to the latter generalization, opiate-induced excitation of hippocampal pyramidal cells was subsequently shown to reflect a disinhibitory phenomenon (i.e., inhibition of inhibitory interneurons) (Zieglgansberger et al., 1979; Madison and Nicoll, 1988).

\section{Relationship between ENK- and $\mathrm{TH}$-immunoreactive processes \\ Ultrastructural associations}

Our finding that $50-60 \%$ of terminals containing ENK-LI form direct synapses on TH-labeled dendrites may represent an underestimation, based on certain methodological limitations: (1) the immunogold method may not be sufficiently sensitive to detect $\mathrm{TH}$ labeling in all processes (particularly in distal dendrites), and (2) lightly ENK-immunoreactive terminals may fail to be detected in single sections if they do not possess a labeled dense-cored vesicle. Thus, the determination of the absolute degree of monosynaptic innervation of $\mathrm{TH}$-labeled processes by ENK-immunoreactive terminals will require further analysis using serial reconstruction. Nevertheless, the findings produced by this single-section analysis, using one of the most sensitive dual-labeling methods to date, represent the first ultrastructural demonstration that a substantial number of terminals containing ENK-LI form direct synaptic contact with TH-labeled neurons in the VTA. The functional importance of this monosynaptic input is further underscored by the observation that multiple ENK-immunoreactive terminals sometimes converged on single TH-labeled dendrites.

The finding that individual ENK-immunoreactive terminals sometimes formed divergent synaptic contacts on multiple dendritic targets (either TH-labeled or unlabeled) implies that opioid peptides may be involved in synchronizing the activity of adjacent cells by providing simultaneous input. This supposition is further supported by the observation that the respective dendrites were often in close apposition to one another. Close membrane appositions between TH-labeled perikarya and dendrites in the VTA have previously been described and proposed to provide a mechanism for synchronized firing in these neurons (Bayer and Pickel, 1990). Although classically defined gap junctions have not yet been identified by electron microscopy in the VTA, dopaminergic neurons in the $\mathrm{SN}$ have been shown to fire in synchrony and to exhibit the physiological properties of electrical coupling (Wilson et al., 1977; Grace and Bunney, 1983).

\section{Correlation with physiological cations of opiates}

Postsynaptic effects. The presence of synapses formed by ENKimmunoreactive terminals on TH-labeled dendrites is at odds with physiological and receptor autoradiographic studies, which seemingly rule out direct $\mu$ - or $\delta$-receptor-mediated effects of opiates on dopaminergic neuronal activity in the VTA (Gysling and Wang, 1983; Dilts and Kalivas, 1989, 1990). It is possible that opiates may act on dopaminergic neurons via a non- $\mu$ non$\delta$ receptor. In this regard, direct excitatory actions of locally applied morphine on VTA dopamine neurons have been described, which are not consistently reversed by naloxone (Gysling and Wang, 1983; Matthews and German, 1984). However, similar effects reported in the rat cerebral cortex in response to 
morphine, but not opioid peptides, appear to result from a nonspecific action of the drug (Stanzione et al., 1989).

Synaptic junctions formed by ENK-immunoreactive terminals on unlabeled dendrites are consistent with physiological reports of direct inhibitory effects of opiate agonists on nondopaminergic neurons in the VTA and adjacent SN (Gysling and Wang, 1983; Lacey et al., 1989). The presence of these synapses also agrees with the localization of $\mu$ - and $\delta$-opiate receptors to nondopaminergic cells (Dilts and Kalivas, 1989, 1990). Thus, the excitatory effects of systemic opiates on dopaminergic neurons may be indirectly mediated through these nondopaminergic neurons, as has been previously suggested (Kelley et al., 1980; Gysling and Wang, 1983; Kalivas and Richardson-Carlson, 1986). However, such a theory presumes that the unlabeled neurons contacted by ENK-immunoreactive terminals contain an inhibitory transmitter and have monosynaptic connections to dopaminergic cells. Such a synaptic organization has been postulated in the $\mathrm{SN}$, but on the basis of physiological, rather than morphological data (Grace and Bunney, 1985). Furthermore, this hypothesis is not entirely consistent with the results of in vitro studies, since inhibitory interneurons should be contained within midbrain slices and mediate an excitatory (disinhibitory) action on dopaminergic cells in response to opiates. This effect has not been observed, at least when examined in the SN (Lacey et al., 1989).

Presynaptic effects. In addition to their direct hyperpolarizing actions on neuronal membranes, opiates have been shown to alter the release of other transmitters through a presynaptic action. This modulatory effect has been demonstrated at several sites within the CNS and PNS and can involve actions on (1) the opioid-originating terminal (autoinhibition or "self-regulation" of colocalized transmitter release) or (2) adjacent processes (lateral inhibition of release) (Jessell and Iversen, 1977; Konishi et al., 1981; Morita and North, 1981; Watt et al., 1984; Lam et al., 1986; Xu et al., 1989). A presynaptic site of action represents an additional mechanism through which opiates can disinhibit cell firing (i.e., by inhibiting the presynaptic release of an inhibitory transmitter). The likelihood that such a mechanism occurs in the VTA is supported by several findings: (1) the direct axonal appositions without intervening astrocytes, which have been presently observed between ENK-immunoreactive terminals and other ENK-labeled and unlabeled terminals, provide a potential substrate for presynaptic regulation of transmitter release; (2) the presence of ENK-LI within dense-cored vesicles in our study suggests a route for release of opioid peptides into the extrasynaptic space (Thureson-Klein and Klein, 1990); (3) $\mu$ - and/or $\delta$-receptors have been localized by light microscopy to striatonigral terminals that contain opioid peptides (AbouKhalil et al., 1984; van der Kooy et al., 1986; see also Kalivas ct al., 1990); and (4) opiate receptors (at least within the striatum) have been localized by electron microscopy to nonsynaptic portions of the plasmalemma, including axoaxonic membrane interfaces (Hamel and Beaudet, 1984).

As mentioned previously, the inhibitory transmitter GABA is one likely candidate for colocalization in opioid-containing afferents to the VTA. Furthermore, enkephalin has been shown to inhibit the release of GABA within the SN and other regions (Watt et al., 1984; Starr, 1985; Lam et al., 1986; Dewar et al., 1987). Thus, it is possible that enkephalin disinhibits dopaminergic cells by diminishing tonic GABA release from its own, or adjacent terminals. This hypothesis is consistent with the combined results of in vivo and in vitro physiological recordings from midbrain neurons, which imply that opiate-induced excitation of dopamine cells requires an afferent input that is tonically active only in the intact brain. The mechanism gains further support from the findings of Kalivas and coworkers that the excitatory action of morphine on VTA dopaminergic neuronal activity can be blocked by the GABA agonist baclofen (Kalivas et al., 1990). The release of other colocalized transmitters besides GABA may also be subject to presynaptic regulation by opioid peptides. The presence of some unlabeled dense-cored vesicles in ENK-immunoreactive terminals in our sample suggests that cotransmitters may include other peptides (Hökfelt et al., 1987; Thureson-Klein and Klein, 1990).

Non-neuronal effects. The observation that dense-cored vesicles containing ENK-LI were apposed to plasmalemmal surfaces in contact with astrocytes suggests the possibility that opioid peptides may be released nonsynaptically in the vicinity of astrocytic processes. This could provide one additional mechanism through which opioid compounds may exert their physiological effects. Opiate receptors have been localized to astrocytes, at least in primary cultures (Rougon et al., 1983; Murphy and Pearce, 1987), suggesting that opioid peptides may regulate the astrocytic uptake and metabolism of other transmitters. Such a mechanism may be particularly relevant for the regulation of GABA transmission, since GABA is both transported into and released from astrocytes (Jaffe and Cuello, 1981; Reynolds and Herschkowitz, 1984). However, whether glial regulation of GABA (or other transmitter) levels can actually be modulated by opioid substances in the VTA requires further investigation.

\section{Conclusions and functional implications}

These studies represent the first ultrastructural demonstration of direct synaptic contact between opioid peptide-containing terminals and dopaminergic and nondopaminergic neurons in the VTA. The findings are consistent with physiological studies describing inhibitory effects of opiates on nondopaminergic midbrain cells. However, the results are discrepant with data from several sources implying indirect effects of these compounds on dopaminergic neurons. The resolution of this discrepancy may depend on future substantiation of certain synaptic, as well as nonconventional (i.e., neuromodulatory), cellular mechanisms for opiate-induced physiological effects.

The modulatory influence of opioid peptides and their receptor agonists on the mesocorticolimbic dopaminergic system has been implicated in the regulation of locomotor and exploratory activity and in the reinforcing properties of opiate drugs. In addition, recent evidence suggests that the well-documented activation of the mesocorticolimbic dopaminergic pathway caused by stress (Thierry et al., 1976; Deutch et al., 1985) is at least partly mediated via release of endogenous opiates into the VTA (Miller ct al., 1984; Kalivas and Abhold, 1987). In addition, daily exposure to either stress or opiate drugs sensitizes the neurochemical and behavioral responses to subsequent exogenously applied opiates (Kalivas, 1985; Kalivas et al., 1986). Thus, the release of endogenous opiates in the VTA and subsequent activation of ascending dopaminergic systems may play a role in stress-induced neuronal plasticity. This speculation has important implications for the pathogenesis of psychotic disorders, which are exacerbated by stressful stimuli and are believed to involve a hyperactivation of forebrain dopaminergic systems (Weinberger, 1987). The present description of multiple morphological substrates for opiate-dopamine interactions in the VTA may guide future studies directed toward a detailed 
understanding of the cellular mechanisms underlying these effects.

\section{References}

Abou-Khalil B, Young AB, Penney JB (1984) Evidence for the presynaptic-localization of opiate binding sites on striatal efferent fibers. Brain Res 323:21-29.

Bayer VE, Pickel VM (1990) Ultrastructural localization of tyrosine hydroxylase in the rat ventral tegmental area: relationship between immunolabcling density and neuronal associations. J Neurosci 10 2996-3013.

Bayer VE, Pickel VM (1991) GABA-labeled terminals form proportionally more synapses with dopaminergic neurons having low densities of tyrosine hydroxylase immunoreactivity in rat ventral tegmental area. Brain Res 559:44-55.

Broekkamp CLE, Phillips AG, Cools AR (1979) Stimulant effects of enkephalin microinjection into the dopaminergic Al0 area. Nature 278:560-562.

Carlin RK, Grab DJ, Cohen RS, Siekovitz P (1980) Isolation and characterization of postsynaptic densities from various brain regions. Enrichment of different types of postsynaptic densities. J Cell Biol 86:831-843.

Chan J, Aoki C, Pickel VM (1990) Optimization of differential immunogold-silver and peroxidase labeling with maintenance of ultrastructure in brain sections before plastic embedding. J Neurosci Methods 33:113-127.

Cuadras J (1989) Non-synaptic release from dense-cored vesicles occurs at all terminal types in crayfish neuropile. Brain Res 477:332335.

Deutch AY, Tam SY, Roth RH (1985) Footshock and conditioned stress increase 3,4-dihydroxyphenylacetic acid (DOPAC) in the ventral tegmental area but not substantia nigra. Brain Res 333:143-146.

Dewar D, Jenner P, Marsden CD (1987) Effects of opioid agonist drugs on the in vitro release of ${ }^{3} \mathrm{H}-\mathrm{GABA},{ }^{3} \mathrm{H}$-dopamine and ${ }^{3} \mathrm{H}-5$ HT from slices of rat globus pallidus. Biochem Pharmacol 36:17381741 .

Di Chiara G, Imperato A (1988) Drugs abused by humans preferentially increase synaptic dopamine concentrations in the mesolimbic system of freely moving rats. Proc Natl Acad Sci USA 85:5274-5278.

Di Figlia M, Aronin N, Martin JB (1982) Light and electron microscopic localization of immunoreactive leu-enkephalin in the monkey basal ganglia. J Neurosci 2:303-320.

Dilts RP, Kalivas PW (1989) Autoradiographic localization of $\mu$-opioid and neurotensin receptors within the mesolimbic dopamine system. Brain Res 488:311-327.

Dilts RP, Kalivas PW (1990) Autoradiographic localization of delta opioid receptors within the mesocorticolimbic dopamine system using radioiodinated [2-D-penicillamine, 5-D-penicillamine] enkephalin (125I-DPDPE). Synapse 6:121-132.

Dores RM, McDonald LK, Steveson TC, Sei CA (1990) The molecular evolution of neuropeptides: prospects for the 90's. Brain Behav Evol 36:80-99.

Fallon JH, Leslie FM (1986) Distribution of dynorphin and enkephalin peptides in the rat brain. J Comp Neurol 249:293-336.

Gaspar P, Berger B, Gay M, Hamon M, Cesselin F, Vigny A, JavoyAgid F, Agid Y (1983) Tyrosine hydroxylase and methionine-enkephalin in the human mesencephalon, immunocytochemical localization and relationships. J Neurol Sci 58:247-267.

Gerfen CR, Young WS III (1988) Distribution of striatonigral and striatopallidal peptidergic neurons in both patch and matrix compartments: an in situ hybridization histochemistry and fluorescent retrograde tracing study. Brain Res 460:161-167.

Grace AA, Bunney BS (1983) Intracellular and extracellular electrophysiology of nigral dopaminergic neurons. III. Evidence for electrical coupling. Neuroscience 10:333-348.

Grace AA, Bunney BS (1985) Opposite effects of striatonigral feedback pathways on midbrain dopamine cell activity. Brain Res 333:271284.

Gray EG (1959) Axosomatic and axo-dendritic synapses of the cerebral cortex: an electron microscopic study. J Anat 93:420-433.

Gysling K, Wang RY (1983) Morphine-induced activation of A10 dopamine neurons in the rat. Brain Res 277:119-127.

Hamel E, Beaudet A (1984) Electron microscopic autoradiographic localization of opioid receptors in rat neostriatum. Nature 312:155157.
Harlan RE, Shivers BD, Romano GJ, Howells RD, Pfaff DW (1987) Localization of preproenkephalin mRNA in the rat brain and spinal cord by in situ hybridization. J Comp Neurol 258:159-184.

Hökfelt T, Millhorn D, Seroogy K, Tsuruo Y, Ceccatelli S, Lindh B, Mcister B, Melander T, Schalling M, Bartfai T, Terenius L (1987) Coexistence of peptides with classical neurotransmitters. Experientia 43:768-780.

Hsu S-M, Raine L, Fanger H (1981) Use of avidin-biotin-peroxidase complex $(\mathrm{ABC})$ in immunoperoxidase techniques: a comparison between $A B C$ and unlabeled antibody (PAP) procedures. J Histochem Cytochem 29:577-580.

Inagaki S, Kubota Y, Kito S (1986) Ultrastructural localization of enkephalin immunoreactivity in the substantia nigra of the monkey. Brain Res 362:171-174.

Jaffe EH, Cuello AC (1981) Neuronal and glial release of [ $\left.{ }^{3} \mathrm{H}\right] \mathrm{GABA}$ from the rat olfactory bulb. J Neurochem 37:1457-1466.

Jessell TM, Iversen LL (1977) Opiate analgesics inhibit substance P release from rat trigeminal nucleus. Nature 268:549-551.

Joh TH, Gcgham C, Rcis DJ (1973) Immunocytochemical demonstration of increased tyrosine hydroxylase protein in sympathetic ganglia and adrenal medulla elicited by reserpine. Proc Natl Acad Sci USA 70:2767-2771.

Johnson RP, Sar M, Stumpf WE (1980) A topographic localization of enkephalin on the dopamine neurons of the rat substantia nigra and ventral tegmental area demonstrated by combined histofluorescenceimmunocytochemistry. Brain Res 194:566-571.

Kalivas PW (1985) Sensitization to repeated enkephalin administration into the ventral tegmental area of the rat. II. Involvement of the mesolimbic dopamine system. J Pharmacol Exp Ther 235:544-550.

Kalivas PW, Abhold R (1987) Enkephalin release into the ventral tegmental area in response to stress: modulation of mesocorticolimbic dopamine. Brain Res 414:339-348.

Kalivas PW, Richardson-Carlson R (1986) Endogenous enkephalin modulation of dopamine neurons in ventral tegmental area. Am J Physiol 251:R243-R249.

Kalivas PW, Widerlov E, Stanley D, Breese G, Prange AJ Jr (1983) Enkephalin action on the mesolimbic system: a dopamine-dependent and a dopamine-independent increase in locomotor activity. J Pharmacol Exp Ther 227:229-237.

Kalivas PW, Richardson-Carlson R, Van Orden G (1986) Cross-sensitization between foot shock stress and enkephalin-induced motor activity. Biol Psychiatry 21:939-950.

Kalivas PW, Duffy P, Eberhardt H (1990) Modulation of A10 dopamine neurons by $\gamma$-aminobutyric acid agonists. J Pharmacol Exp Ther 253:858-866.

Kelley AE, Stinus L, Iversen SD (1980) Interactions between D-alamel-enkephalin, A10 dopaminergic neurones, and spontaneous behavior in the rat. Behav Brain Res 1:3-24.

Khachaturian H, Lewis ME, Hollt V, Watson SJ (1983a) Telencephalic enkephalinergic systems in the rat brain. J Neurosci 3:844-855.

Khachaturian H, Lewis ME, Watson SJ (1983b) Enkephalin systems in diencephalon and brainstem of the rat. J Comp Neurol 220:310 320 .

Kim HS, Iyengar S, Wood PL (1986) Opiate actions on mesocortical dopaminc metabolism in the rat. Life Sci 39:2033-2036.

Klein RL, Wilson SP, Dzielak DJ, Yang WH, Viveros OH (1982) Opioid peptides and noradrenaline co-exist in large dense-cored vesicles from sympathetic nerve. Neuroscience 7:2255-2261.

Konishi S, Tsunoo A, Otsuka M (1981) Enkephalin as a transmitter for presynaptic inhibition in sympathetic ganglia. Nature 294:80-82.

Koob GF, Bloom FE (1988) Cellular and molecular mechanisms of drug dependence. Science 242:715-723.

Lacey MG, Mercuri NB, North RA (1989) Two cell types in rat substantia nigra zona compacta distinguished by membrane properties and the action of dopamine and opioids. J Neurosci 9:1233-1241.

Lam DMK, Su YYT, Watt CB (1986) The self-regulating synapse: a functional role for the co-existence of neuroactive substances. Brain Res Rev 11:249-257.

Larsson LI (1981) A novel immunocytochemical model system for specificity and sensitivity screening of antisera against multiple antigens. J Histochem Cytochem 29:408-410.

Latimer LG, Duffy P, Kalivas PW (1987) Mu opioid receptor involvement in enkephalin activation of dopamine neurons in the ventral tegmental area. J Pharmacol Exp Ther 241:328-337.

Leranth C, Pickel VM (1989) Electron microscopic pre-embedding 
double immunostaining methods. In: Tract-tracing methods 2 , Recent progress (Heimer L, Zaborsky L, eds), pp 129-172. New York: Plenum.

Madison DV, Nicoll RA (1988) Enkephalin hyperpolarizes interneurones in the rat hippocampus. J Physiol (Lond) 398:123-130.

Mansour A, Khachaturian H, Lewis ME, Akil H, Watson SJ (1987) Autoradiographic differentiation of $\mathrm{mu}$, delta, and kappa opioid receptors in the rat forebrain and midbrain. J Neurosci 7:2445-2464.

Matthews RT, German DC (1984) Electrophysiological evidence for excitation of rat ventral tegmental area dopamine neurons by morphine. Neuroscience 11:617-625.

Miller JD, Speciale SG, McMillen BA, German DC (1984) Naloxone antagonism of stress-induced augmentation of frontal cortex dopamine metabolism. Eur J Pharmacol 98:437-439.

Milner TM, Pickel VM, Reis DJ (1989) Ultrastructural basis for interactions between central opioids and catecholamines. I. Rostral ventrolateral medulla. J Neurosci 9:2114-2130.

Morita K, North RA (1981) Opiates and enkephalin reduce the excitability of neuronal processes. Neuroscience 6:1943-1951.

Murphy S, Pearce B (1987) Functional receptors for neurotransmitters on astroglial cells. Neuroscience 22:381-394.

Navone F, Greengard P, De Camilli P (1984) Synapsin I in nerve terminals: selective association with small synaptic vesicles. Science 226:1209-1211.

Nicoll RA, Siggins GR, Ling N, Bloom FE, Guillemin R (1977) Neuronal actions of endorphins and enkephalins among brain regions: a comparative microiontophoretic study. Proc Natl Acad Sci USA 74: 2584-2588.

Pelletier G, Leclerc R (1979) Localization of leu-enkephalin in dense core vesicles of axon terminals. Neurosci Lett 12:159-163.

Peters A, Palay SL, Webster HD (1991) The fine structure of the nervous system. Neurons and their supporting cells. New York: Oxford UP.

Phillipson OT (1979) Afferent projections to the ventral tegmental area of Tsai and interfascicular nucleus: a horseradish peroxidase study in the rat. J Comp Neurol 187:117-144.

Pickel VM, Sumal KK, Beckley SC, Miller RJ, Reis DJ (1980) Immunocytochenical localization of enkephalin in the neostriatum of rat brain: a light and electron microscopic study. J Comp Neurol 189: $721-740$.

Pickel VM, Chan J, Sesack SR (1991) Monosynaptic input to dopaminergic neurons in rat substantia nigra and ventral tegmental area from dynorphin-immunoreactive terminals. Neurosci Abstr 17:718.

Reiner A, Anderson K (1990) The patterns of neurotransmitter and neuropeptide co-occurrence among striatal projection neurons: conclusions hased on recent findings. Brain Res Rev 15:251-265.

Reynolds R, Herschkowitz N (1984) Uptake of [ $\left.{ }^{3} \mathrm{H}\right] \mathrm{GABA}$ by oligodendrocytes in dissociated brain cell culture: a combined autoradiographic and immunocytochemical study. Brain Res 322:17-31.

Rougon G, Noble M, Mudge AW (1983) Neuropeptides modulate the $\beta$-adrenergic response of purified astrocytes in vitro. Nature 305:715717.

Sesack SR, Pickel VM (1990) Synaptic input from opioid terminals to dopaminergic and non-dopaminergic neurons in the ventral tegmental area of the rat. Eur J Pharmacol 183:208-209.

Shippenberg TS, Herz A (1987) Place preference conditioning reveals the involvement of $D_{1}$-dopamine receptors in the motivational properties of $\mu$ - and $\kappa$-opioid agonists. Brain Res 436:169-172.

Somogyi P, Priestly JV, Cuello AC, Smith AD, Takagi H (1982) Syn- aptic connections of enkephalin-immunoreactive nerve terminals in the neostriatum: a correlated light and electron microscopic study. J Neurocytol 11:779-807.

Stanzione P, Stefani A, Calabresi P, Mercuri NB, Bernardi G (1989) Met- and leu-enkephalins inhibit rat cortical neurons intracellularly recorded in vivo while morphine excites them: evidence for naloxonesensitive and naloxone-insensitive effects. Exp Brain Res 77:302-308.

Starr MS (1985) Multiple opiate receptors may be involved in suppressing $\gamma$-aminobutyrate release in substantia nigra. Life Sci 37 : 2249-2255.

Stinus L, Koob GF, Ling N, Bloom FE, LeMoal M (1980) Locomotor activation induced by infusion of endorphins into the ventral tegmental area: evidence for opiate-dopamine interactions. Proc Natl Acad Sci USA 77:2323-2327.

Sumal KK, Pickel VM, Miller RJ, Reis DJ (1982) Enkephalin-containing neurons in substantia gelatinosa of spinal trigeminal complex: ultrastructure and synaptic interaction with primary sensory afferents. Brain Res 248:223-236

Thierry AM, Tassin JP, Blanc G, Glowinski J (1976) Selective activation of the mesocortical DA system by stress. Nature $263: 242-244$.

Thureson-Klein AK, Klein RL (1990) Exocytosis from neuronal large dense-cored vesicles. Int Rev Cytol 121:67-126.

Vaccarino FJ, Amalric M, Swerdlow NR, Koob GF (1986) Blockade of amphetamine but not opiate-induced locomotion following antagonism of dopamine function in the rat. Pharmacol Biochem Behav 24:61-65.

van der Kooy D, Weinreich P, Nagy JI (1986) Dopamine and opiate receptors: localization in the striatum and evidence for their axoplasmic transport in the nigrostriatal and striatonigral pathways. Neuroscience 19:139-146.

Van Ree JM, Ramsey N (1987) The dopamine hypothesis of opiate reward challenged. Fur J Pharmacol 134:239-243.

Watt CB, Su YYT, Lam DMK (1984) Interaction between enkephalin and GABA in avian retina. Nature 311:761-763.

Weinberger DR (1987) Implications of normal brain development for the pathogenesis of schizophrenia. Arch Gen Psychiatry 44:660-669.

Williams JT, Egan TM, North RA (1982) Enkephalin opens potassium channels on mammalian central neurones. Nature 299:74-77.

Wilson CJ, Young SJ, Groves PM (1977) Statistical properties of neuronal spike trains in the substantia nigra: cell types and their interactions. Brain Res 136:243-260.

Wise RA (1989) Opiate reward: sites and substrates. Neurosci Biobehav Rev 13:129-133.

Xu H, Smolens I, Gintzler AR (1989) Opioids can enhance and inhibit the electrically evoked release of methionine-enkephalin. Brain Res 504:36-42

Zamir N, Palkovits M, Weber E, Mezey E, Brownstein M (1984) A dynorphinergic pathway of leu-enkephalin production in rat substantia nigra. Nature 307:643-645.

Zamir N, Palkovits M, Brownstein M (1985) Distribution of immunoreactive met-enkephalin-arg ${ }^{6}-\mathrm{gly}^{7}-\mathrm{leu}^{8}$ and leu-enkephalin in discrete regions of the rat brain. Brain Res 326:1-8.

Zhu PC, Thureson-Klein A, Klein RL (1986) Exocytosis from large dense cored vesicles outside the active synaptic zones of terminals within the trigeminal subnucleus caudalis: a possible mechanism for neuropeptide release. Neuroscience 19:43-54.

Zieglgansberger W, French ED, Siggins GR, Bloom FE (1979) Opioid peptides may excite hippocampal pyramidal neurons by inhibiting adjacent inhibitory interneurons. Science 205:415-417. 